\title{
A Novel Approach for Therapeutic Use of Phenolics for the Prevention and Management of Mental Illness
}

\author{
Vincent Candrawinata* \\ Department of Mental Health, Australia
}

*Corresponding author: Vincent Candrawinata, Department of Mental Health, Australia.

\section{Short Communication}

The prevalence of mental illness permanently increases. In some countries, the statistics are staggering, one in five people in Australia between the age of $16-85$ experience a mental illness. Conventional treatments often involve antipsychotic drugs, especially stimulants or mood regulators which have reported significant adverse effects, another approach utilising more natural compounds may help in promoting mental health and potentially the prevention and management of mental illness.

Phenolics are naturally occurring compound found in plants. Phenolics are powerful free radical 'scavengers' because of the numbers of hydroxyl groups in their chemical structures. The number of hydroxyl groups correspond to a compound's ability to neutralise free radicals. The hydroxyl groups are essentially the electrons which are donated to free radical molecules to stop them stealing electrons from human body's cells. However, in addition to their antioxidant property, there are other biomodulating properties that may be linked to mental health.

Among the arrays of disorders in the pathogenesis of mental illness, oxidative stress, increased inflammatory processes, defects in neurogenesis and synaptic plasticity, mitochondrial dysfunction, and redox imbalance are regularly observed. Although there has not been a report on the direct interference between phenolics and the pathophysiology of mental illness such as schizophrenia, phenolics are potentially beneficial in modulating mental health including brain plasticity, behaviour, mood, depression, and cognition.

One of the most challenging factors of phenolics as treatment is its bioavailability. The low bioavailability and transformation of phenolics in vivo to different derivatives lead to their low direct antioxidant activity in comparison to other low-molecular weight antioxidants, such as vitamins C. The poor absorption is not only rendering these phenolics useless in the body, the unabsorbed phenolics could cause harm to the body's system. High concentration of these unusable compounds adds to the workload of kidney and liver, which over a long period could have detrimental effects to the organs.

A simple example is that when someone consumes too many blueberries or drink beetroot or carrot juice, they would notice changes in their urine colour or sometimes experiencing diarrhoea. This is because human body can only absorb a tiny fraction of the phenolics at once and it has to flush out the unused portion. Based on the chemical structure and conformation, phenolics should perform better in the fight against free radicals. Vitamin $\mathrm{C}$, with less hydroxyl groups, after collecting a free radical, can become unstable-this is known as pro-oxidation and can do more harm than good.

Pro-oxidation, as the name suggests, is the polar opposite of the functions of antioxidant. Pro-oxidation happens because when an antioxidant molecule gives up its electron to neutralise a free radical molecule, it becomes a free radical itself. Therefore, although it neutralises one free radical molecule, it is now starting an oxidation chain reaction of its own. There are many pathways in which phenolics may positively influence the health of human neurological system. The anti-inflammatory effects of phenolics in cerebral tissue can be achieved though bonding with neural receptors. Flavonoid-induced receptor stimulation can modulate active state of different kinases, such as the mitogen-activated protein (MAP) kinase, phosphoinositide-3-(PI3) kinase, nuclear factor-kappa B, and protein kinase C (PKC) pathways. Phenolics can thus influence differentiation and apoptosis, cell survival (inhibition of apoptosis), inflammatory response, learning and memory, and reduction of amyloid plaque formation. Further antiinflammatory property can be demonstrated through the activation of glial cells in brain, which macrophages are found, which then the 
inhibition of the inflammation rate is mediated by macrophages through the reduction of proinflammatory cytokines (IL-1 beta, TNF-alpha) formation.

The neuroprotection of phenolics can contribute to better blood flow to the brain via stimulating the NO formation in the endothelium while inhibiting of platelet aggregation. Nevertheless, the question remains, how and in which form can phenolics be available in our system and modulate its function? A new breed of dietary phenolics are emerging, accessed with a breakthrough technology that just uses water to extract and activate the phenolics. This technology gives the phenolics the synergy, absorbability and bioavailability that are the keys in achieving therapeutic effects in a biological system.

The unprecedented potency of the antioxidant is achieved through new natural extraction technology which involves no chemical solvent at any stage of the process. This is achieved by manipulating the behaviour of water molecules to extract and activate the phenolics, resulting in a highly water-soluble antioxidant which has better bioavailability than those which are made either synthetically in a laboratory or extracted from natural foods using chemicals (methanol, acetone or ethanol), making them very difficult to break down and absorb and could potentially cause more harm than good.

It is time to understand that phenolics, when properly sourced, extracted and activated, do work synergistically with the body to deliver promising therapeutic effects to promote mental health, preventing and managing mental illness.

https://www.blackdoginstitute.org.au/docs/default-source/ factsheets/facts_figures.pdf?sfvrsn=8

\section{Acknowledgement}

None.

\section{Conflict of Interest}

No conflict of interest. 